\title{
La protección de las personas con discapacidad en el derecho italiano: interdizione, inabilitazione y amministrazione di sostegno
}

\author{
The protection of people with disabilities in Italian law: interdizione, \\ inabilitazione and amministrazione di sostegno
}

\begin{abstract}
Resumen
La protección de las personas con discapacidad en el derecho italiano se lleva a cabo a través de tres instrumentos: la interdizione, la inabilitazione y la amministrazione di sostegno. La introducción de esta última figura en el año 2004 supuso un importante avance, no solo en el derecho italiano, sino también a nivel comparado, anticipando la que se convertiría en la nueva tendencia en materia de protección de las personas con discapacidad, y sirviendo de inspiración a los legisladores de otros estados, entre ellos el nuestro. Ahora bien, la amministrazione di sostegno ha planteado desde el primer momento importantes problemas de delimitación con las tradicionales figuras de la interdizione y la inabilitazione, tanto en relación con su contenido como a los presupuestos en los que entran en juego. Además, la competencia para establecer una y otras corresponde a distintos órganos, lo que ha dado lugar a algunas dificultades de coordinación entre los mismos. Todo ello ha requerido una amplia labor interpretativa por parte de la jurisprudencia y doctrina italianas, que es objeto de estudio en el presente escrito.
\end{abstract}

\section{Palabras clave}

Discapacidad, interdizione, inabilitazione, amministrazione di sostegno, tutela, curatela.

\begin{abstract}
The protection of people with disabilities in Italian law is carried out through three instruments: interdizione, inabilitazione and amministrazione di sostegno. The introduction of the latter figure in $\mathbf{2 0 0 4}$ was an important step forward, not only in Italian law, but also on a comparative level, anticipating what would become the new trend in the protection of disabled people, and inspiring legislators in other States, including our own. However, from the outset, the amministrazione di sostegno has raised major problems of demarcation with the traditional figures of interdizione and inabilitazione, both in relation to their content and to the budgets in which they come into play. In addition, the competence to establish one and other corresponds to different courts, which has led to some difficulties of coordination between them. All of this has required extensive interpretative work by the Italian jurisprudence and doctrine, which is the subject of this paper.
\end{abstract}

\section{Keywords}

Disability, incapacitation, disabling, supporting administration, guardianship, curatorship.

\author{
Javier Martínez Calvo \\ $<$ jjaviermartinezcalvo@gmail. \\ com> \\ Universidad de Zaragoza. España
}

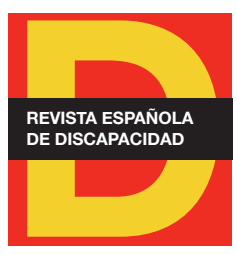

Para citar:

Martínez, J. (2020). "La protección de las personas con discapacidad en el derecho italiano: interdizione, inabilitazione y amministrazione di sostegno". Revista Española de Discapacidad, 8(I), pp. 43-61.

Doi: <https://doi.org/10.5569/23405104.08.01.03>

Fecha de recepción: 14-04-2019 Fecha de aceptación: 06-05-2020 


\section{El sistema italiano de protección de las personas privadas de autonomía y su contribución al cambio de paradigma en materia de discapacidad ${ }^{1}$}

El ordenamiento jurídico italiano prevé tres instrumentos para la protección de las personas privadas de autonomía: la interdizione - que se materializa en la figura de la tutela -, la inabilitazione - que se materializa en la curatela - (Rocchio y Liserre, 2017: 104-105) y la amministrazione di sostegno -en español: "administración de apoyo"- - Este último instrumento fue introducido en el derecho italiano mediante la Ley n. 6, de 9 de enero de 2004, cuyo artículo 3.1 modificó los artículos 404 y siguientes del Codice Civile, que recogen el régimen jurídico de dicha figura.

La introducción de la amministrazione di sostegno supuso una importante novedad no sólo en el derecho italiano, sino también en el marco del derecho comparado, pues rompió con el que venía siendo el sistema tradicional de protección de las personas con discapacidad - caracterizado por la sustitución en la toma de decisiones - y creó un sistema basado en la voluntad y las preferencias de la persona con discapacidad, que, como regla general, pasa a ser la encargada de tomar sus propias decisiones.

Con ello, el legislador italiano se adelantó a la célebre Convención sobre los derechos de las personas con discapacidad, hecha en Nueva York el 13 de diciembre de 2006, que, de acuerdo a lo dispuesto en su artículo primero, tiene como propósito que las personas con discapacidad puedan participar plena y efectivamente en la sociedad, en igualdad de condiciones con las demás; y que en su artículo 3 a) establece, como principio general, el respeto a la autonomía individual, incluida la libertad de tomar las propias decisiones. Especialmente revelador resulta su artículo 12, que lleva por rúbrica "Igual reconocimiento como persona ante la ley", y que, entre otros aspectos, dispone que las personas con discapacidad tienen capacidad jurídica en igualdad de condiciones con las demás en todos los aspectos de la vida, y obliga a los Estados parte a adoptar las medidas pertinentes para proporcionar acceso a las personas con discapacidad al apoyo que puedan necesitar en el ejercicio de su capacidad jurídica - una obligación que está prevista también en el artículo 4 de la Convención-.

Aunque resultaría aventurado aseverar que la ley italiana haya sido la inspiradora de dicha Convención - pues es resultado de una corriente ideológica que se venía gestando ya desde largo tiempo atrás -, cabe reconocerle el mérito de haber supuesto una de las primeras manifestaciones de la que parece haberse convertido en la nueva tendencia en materia de protección de las personas con discapacidad, caracterizada por una ampliación de su libre autonomía de la voluntad. De hecho, pese a ser anterior a la Convención, se encuentra en perfecta sintonía con ella. Como señala Vivas Tesón, "La Ley 6/2004, pese a ser de fecha anterior - reparemos en que la Ley es de 2004 pero su verdadera gestación se produjo muchos años antesa la Convención ONU - ratificada por Italia, recuérdese, mediante la reciente Ley 18/2009, de 3 de marzo-, es muy respetuosa con los principios, valores y modos de concebir la discapacidad que ésta, posteriormente, recogería, en especial, en lo que se refiere a la regulación de la administración de apoyo, que se ajusta

1. Este trabajo ha sido realizado en el marco del Grupo Consolidado de Investigación del Gobierno de Aragón "Ius Familiae", IP. Carlos Martínez de Aguirre Aldaz; y del Proyecto de Investigación MINECO: DER2016-75342-R "Prospectiva sobre el ejercicio de la capacidad: la interrelación entre las reformas legales en materia de discapacidad y menores", IIPP. Sofía de Salas Murillo/Ma Victoria Mayor del Hoyo. Ha sido elaborado con ocasión de la realización de una estancia de investigación en Bologna (Italia), cofinanciada por la Universidad de Zaragoza, Fundación Bancaria Ibercaja y Fundación CAl (ref. CH 11/17) y el Ministerio de Educación (beca Erasmus +). 
perfectamente, en su ratio y en sus presupuestos, al modelo social de discapacidad que proclama el citado instrumento normativo internacional" (Vivas, 2012: 62). Ello no obsta para que haya alguna cuestión que quizá podría revisarse para una mejor adaptación de la norma italiana a la Convención -ej. recogiendo la posibilidad de establecer medidas preventivas por parte del interesado en previsión de una futura necesidad de apoyo, como posteriormente veremos-.

Por lo demás, resulta bastante probable que la normativa italiana haya servido de inspiración a los legisladores de otros Estados, y, entre ellos, a nuestro propio legislador, pues como vamos a ver, el nuevo anteproyecto de ley por la que se reforma la legislación civil y procesal en materia de discapacidad, aprobado por el gobierno el 21 de septiembre de 2018, tiene importantes puntos de conexión con la normativa italiana.

El mayor problema que ha provocado en la práctica la introducción de la amministrazione di sostegno en el derecho italiano tiene que ver con su delimitación con las tradicionales figuras de la interdizione y la inabilitazione. El objetivo principal de este trabajo es precisamente delimitar los tres instrumentos que recoge el derecho italiano para la protección de las personas con discapacidad, explicando las diferencias y similitudes existentes entre ellos. En concreto, se abordarán los presupuestos y contenido de cada una de las tres figuras.

Así mismo, será objeto de análisis la cuestión competencial, en la que también se han producido algunos problemas como consecuencia de la introducción de la amministrazione di sostegno. Y es que, el órgano encargado de adoptar esta figura no es el mismo que el competente para establecer la interdizione y la inabilitazione, lo que ha dado lugar a algunas dificultades de coordinación entre los distintos órganos judiciales.

A tal fin, se llevará a cabo un estudio de la jurisprudencia y doctrina italiana, ya que ello nos permitirá comprender mejor la normativa, pues como veremos, en ocasiones resulta confusa, lo que ha dado lugar a importantes problemas interpretativos.

Finalmente, se abordará la incidencia que ha tenido la normativa italiana en la confección del anteproyecto de ley por la que se reforma la legislación civil y procesal en materia de discapacidad. A tal efecto, se ahondará en las grandes similitudes que presentan ambas normativas, pero también en algunas de las principales diferencias existentes entre una y otra.

\section{Delimitación entre interdizione, inabilitazione y amministrazione di sostegno}

Como he anticipado, la Ley n. 6, de 9 de enero de 2004, introdujo en el ordenamiento jurídico italiano la figura de la amministrazione di sostegno. En un primer momento hubo algún autor que consideró que, con la entrada en vigor de dicha norma, habían quedado tácitamente derogadas la interdizione y la inabilitazione (Buffone, 2013: 8). No obstante, la Corte Costituzionale italiana, en la primera resolución en la que se pronunció acerca de esta cuestión, afirmó que tanto la interdizione como la inabilitazione subsistían y podían ser objeto de establecimiento por parte de los tribunales (Corte Cost., 9 dicembre 2005). Así, se configuró un sistema de protección de las personas con discapacidad articulado a través de tres instrumentos diferentes: la interdizione, la inabilitazione y la amministrazione di sostegno. 
Pero entonces, se plantearon dos importantes problemas: cuál es la diferencia entre los mencionados instrumentos y cuándo procede el establecimiento de cada uno de ellos. Junto a ellos, como he señalado, también surgieron algunos problemas competenciales. A continuación, paso a tratar por separado las tres cuestiones.

a) Concepto y contenido de la interdizione, la inabilitazione y la amministrazione di sostegno: diferencias y puntos de conexión

La jurisprudencia y doctrina italianas han coincidido en señalar que la principal diferencia que mantiene la amministrazione di sostegno con las tradicionales figuras de la interdizione y la inabilitazione es que esta se presenta como una institución dotada de una enorme flexibilidad (Paradiso, 2005: 2; Marcoz, 2005: 6; Balestra, 2005:1; Curatola, 2006: 2-4; Serrao, 2006: 6; Lavedini, 2007: 1; Cocuccio, 2010: 4; Giorgianni, 2013 : 1; Rocchio y Liserre, 2017: 106), cuyo objetivo es sacrificar lo menos posible la capacidad del beneficiario para actuar en el tráfico jurídico (Corte Cost., 9 dicembre 2005, Cass., 26 ottobre 2011 y Cass., 11 settembre 2015; Eramo, 2004: 1-2; Bembo, 2005: 1; Curatola, 2006: 7). Y es que, el beneficiario de la amministrazione di sostegno conserva la capacidad para realizar por sí mismo todos los actos que, de acuerdo a lo previsto en el Decreto di nomina - que es la resolución por la que se establece esta figura-, no requieran de la representación o asistencia del amministratore di sostegno - artículo 409.1 del Codice Civile - ${ }^{2}$. El amministratore di sostegno (que podrá ser una persona, o excepcionalmente dos: Trib. Genova, 17 dicembre 2015³), por su parte, representa o asiste al beneficiario exclusivamente en aquellos actos que consten en el Decreto di nomina (Eramo, 2004: 4; Bembo, 2005: 4; Curatola, 2006: 1-2; Giorgianni, 2013: 2). Además, el beneficiario de la amministrazione di sostegno puede realizar autónomamente todos los actos que tengan por objeto la satisfacción de las necesidades básicas de la vida cotidiana - artículo 409.2 del Codice Civile-4 ${ }^{4}$.

Por contra, la interdizione y la inabilitazione tienen un contenido más rígido que el de la amministrazione di sostegno, derivado de la aplicación de las reglas previstas para la tutela de los menores de edad no emancipados y para la curatela de los menores emancipados, respectivamente - artículo 424.1 del Codice Civile italiano- ${ }^{5}$. En este sentido, el artículo 357 del Codice Civile, referido a la tutela sobre el menor de edad, prevé que el tutor represente a este en todos los actos de la vida civil6; y, el artículo 394 del Codice Civile, relativo al ejercicio de la curatela sobre el menor emancipado, establece que este sólo puede llevar a cabo por sí mismo aquellos actos que no excedan de la ordinaria administración, necesitando la asistencia del curador para el resto de actos ${ }^{7}$.

\footnotetext{
2. Il beneficiario conserva la capacità di agire per tutti gli atti che non richiedono la rappresentanza esclusiva o l'assistenza necessaria dell'amministratore di sostegno.

3. En dicho pronunciamiento, el Tribunale admite la posibilidad de que en los supuestos más complejos, o en los que exista un alto riesgo de que puedan darse conflictos de intereses entre el beneficiario y el amministratore di sostegno, se nombre a dos personas distintas para el ejercicio de dicha función. En el caso en cuestión se establece un régimen de amministratore di sostegno en favor de una persona que tiene autismo y, debido a que únicamente es capaz de expresar su voluntad ante sus progenitores, se considera que ambos deben desempeñar conjuntamente esta función.

4. Il beneficiario dell'amministrazione di sostegno può in ogni caso compiere gli atti necessari a soddisfare le esigenze della propria vita quotidiana. 5. Le disposizioni sulla tutela dei minori [343 ss.] e quelle sulla curatela dei minori emancipati [390 ss.] si applicano rispettivamente alla tutela degli interdetti e alla curatela degli inabilitati.

6. Il tutore ha la cura della persona del minore, lo rappresenta in tutti gli atti civili e ne amministra i beni.

7. L'emancipazione conferisce al minore la capacità di compiere gli atti che non eccedono l'ordinaria amministrazione(...)Per gli altri atti eccedenti l'ordinaria amministrazione, oltre il consenso del curatore, è necessaria l'autorizzazione del giudice tutelare"
} 
No se puede perder de vista que tras la entrada en vigor de la Ley n. 6, de 9 de enero de 2004 -artículo 9- se han suavizado dichas previsiones, al admitirse que la sentencia de interdizione o inabilitazione pueda establecer que algunos de los actos de ordinaria administración puedan ser llevados a cabo por el propio interdetto - bien por sí mismo o bien con la asistencia del tutor - y que algunos de los actos de extraordinaria administración puedan ser realizados por el inabilitato sin la asistencia del curador —artículo 427.1 del Codice Civile ${ }^{8}$. No obstante, lo cierto es que la interdizione y la inabilitazione continúan presentando un contenido bastante menos flexible que el de la amministrazione di sostegno (Mascolo y Marcoz, 2005: 1; Paradiso, 2005: 5; Cocuccio, 2010: 2-4; lurilli, 2017: 5). Hay que tener en cuenta que este último instrumento proporciona a las personas con discapacidad un sistema que podrá consistir bien en la representación o bien en la asistencia - en función del acto de que se trate- (Trib. Bologna, 2 gennaio 2006 y Trib. Pinerolo, 13 dicembre 2005; Vitulo, 2004: 3; Marcoz, 2005: 6; Curatola, 2006: 4; y Giorgianni, 2013: 2) y que se limita únicamente a aquellos actos que no pueden llevar a cabo por sí mismas. Es por ello que la doctrina ha afirmado que la amministrazione di sostegno consiste en crear un proyecto personalizado o un "traje a medida" ajustado a las necesidades específicas de cada persona (Di Marzio, 2005: 2; Masoni, 2007: 4; Cocuccio, 2010: 4; Cordiano, 2011: 2; Masoni, 2016: 4).

Como adelantaba, el contenido de la amministrazione di sostegno será el previsto en cada caso en el Decreto di nomina (Cordiano, 2011: 1), que, por tanto, constituye la fuente fundamental en la materia. De hecho, a diferencia de lo que ocurre en los supuestos de interdizione o inabilitazione, el régimen legal supletorio que el legislador ha previsto para la amministrazione di sostegno es muy reducido (Mascolo y Marcoz, 2005: 1; Marcoz, 2005: 6; y Rocchio y Liserre, 2017: 106).

Sin perjuicio de todo lo anterior, también existen algunos puntos de conexión entre las tres figuras. A modo de ejemplo, el Codice Civile prevé expresamente que algunas de las prescripciones relativas al tutor se apliquen también al amministratore di sostegno - artículo 411.1 y 2 del Codice Civile ${ }^{9}{ }^{10}$. Así, al amministratore di sostegno se le aplican las mismas causas de incapacidad que al tutor - artículo 411.1 del Codice Civile ${ }^{11}$ en relación al artículo 350 del mismo texto ${ }^{12}-$, por lo que no podrán desempeñar este cargo las siguientes personas: aquellos que no cuenten con la libre administración del propio patrimonio, aquellos que hayan sido excluidos para el ejercicio del cargo por la última persona que hubiera ejercido la responsabilidad parental sobre el beneficiario, aquellos que tengan un litigio contra el beneficiario por el cual este o su patri-

8. Nella sentenza che pronuncia l'interdizione o l'inabilitazione, o in successivi provvedimenti dell'autorità giudiziaria, può stabilirsi che taluni atti di ordinaria amministrazione possano essere compiuti dall'interdetto senza l'intervento ovvero con l'assistenza del tutore, o che taluni atti eccedenti l'ordinaria amministrazione possano essere compiuti dall'inabilitato senza l'assistenza del curatore.

9. Si applicano all'amministratore di sostegno, in quanto compatibili, le disposizioni di cui agli articoli da 349 a 353 e da 374 a 388 (...)" "All'amministratore di sostegno si applicano altresi, in quanto compatibili, le disposizioni degli articoli 596, 599 e 779.

10. Incluso se admite que el juez disponga que determinados efectos o limitaciones previstos para el interdetto o el inabilitato puedan extenderse al beneficiario de la amministrazione di sostegno - artículo 411.4 del Codice Civile: Il giudice tutelare, nel provvedimento con il quale nomina l'amministratore di sostegno, o successivamente, può disporre che determinati effetti, limitazioni o decadenze, previsti da disposizioni di legge per l'interdetto o l'inabilitato, si estendano al beneficiario dell'amministrazione di sostegno, avuto riguardo all'interesse del medesimo ed a quello tutelato dalle predette disposizioni. Il provvedimento è assunto con decreto motivato a seguito di ricorso che può essere presentato anche dal beneficiario direttamente-. Ahora bien, salvo en los supuestos legalmente previstos, la jurisprudencia se ha mostrado contraria a que puedan extenderse analógicamente a la amministrazione di sostegno las normas previstas en sede de interdizione e inabilitazione - vid. Cass., 16 novembre 2007: «resta indubbio che, in seno agli artt. 404 ss. c.c., è stato disegnato un procedimento dotato di una sua autonomia e peculiarità, che esclude l'applicazione in via di interpretazione estensiva di norme diverse da quelle espressamente richiamate»-

11. Si applicano all'amministratore di sostegno, in quanto compatibili, le disposizioni di cui agli articoli da 349 a $353(. .$.$) .$

12. Non possono essere nominati tutori e, se sono stati nominati, devono cessare dall'ufficio: 1) coloro che non hanno la libera amministrazione del proprio patrimonio; 2) coloro che sono stati esclusi dalla tutela per disposizione scritta del genitore il quale per ultimo ha esercitato la responsabilità genitoriale; 3) coloro che hanno o sono per avere o dei quali gli ascendenti, i discendenti o il coniuge hanno o sono per avere col minore una lite, per effetto della quale può essere pregiudicato lo stato del minore o una parte notevole del patrimonio di lui; 4) coloro che sono incorsi nella perdita della responsabilità genitoriale o nella decadenza da essa, o sono stati rimossi da altra tutela; 5) il fallito che non è stato cancellato dal registro dei falliti. 
monio puedan verse perjudicados, aquellos que hayan sido privados de la patria potestad o removidos de la tutela y aquellos que hubieran sido declarados en concurso de acreedores.

Así mismo, al amministratore di sostegno se le prohíbe llevar a cabo los mismos actos que tiene prohibido realizar el tutor: adquirir o recibir en arrendamiento bienes o derechos del beneficiario, ya sea directamente o a través de un tercero, o convertirse en cesionario de créditos contra el beneficiario - artículo 411.1 del Codice Civile $^{13}$ en relación al artículo 378 del mismo texto ${ }^{14}-$.

Además, al igual que el tutor, precisará de autorización judicial para llevar a cabo determinados actos: adquirir bienes - con la salvedad de aquellos bienes muebles que resulten necesarios para el uso del beneficiario, para la economía doméstica o para la administración del patrimonio-, contraer obligaciones —salvo las relativas a los gastos necesarios para el mantenimiento del beneficiario o la administración de su patrimonio-, aceptar o renunciar a herencias, aceptar donaciones o legados sujetos a cargas o condiciones, suscribir contratos de arrendamiento sobre bienes inmuebles con una duración superior a nueve años, promover procesos judiciales en nombre del beneficiario, vender bienes - excepto los frutos y bienes muebles que pudieran deteriorarse - o constituir un derecho de prenda o hipoteca sobre los mismos —artículo 411.1 del Codice Civile ${ }^{15}$ en relación a los artículos $374^{16}$ y $375^{17}$ del mismo texto-.

\section{b) Presupuestos de la interdizione, la inabilitazione y la amministrazione di sostegno}

Los presupuestos de la amministrazione di sostegno están previstos en el artículo 404 del Codice Civile italiano, que señala que podrá ser establecida en favor de una persona que, por razón de una enfermedad o discapacidad física o psíquica ${ }^{18}$, se encuentre imposibilitada, aun cuando sea parcial o temporalmente, para la gestión de sus propios intereses ${ }^{19}$. Del tenor literal del precepto puede deducirse que se requieren dos presupuestos cumulativos para poder activar la amministrazione di sostegno (Curatola, 2006: 3; y Masoni, 2009: 1): 1) el hecho de tener una enfermedad o discapacidad, que podrá ser psíquica o física ${ }^{20}$ y temporal o

13. Si applicano all'amministratore di sostegno, in quanto compatibili, le disposizioni di cui agli articoli (...) da 374 a 388.

14. II tutore e il protutore non possono, neppure all'asta pubblica, rendersi acquirenti direttamente o per interposta persona dei beni e dei diritti del minore. Non possono prendere in locazione i beni del minore senza l'autorizzazione e le cautele fissate dal giudice tutelare. Gli atti compiuti in violazione di questi divieti possono essere annullati su istanza delle persone indicate nell'articolo precedente, ad eccezione del tutore e del protutore che li hanno compiuti. II tutore e il protutore non possono neppure diventare cessionari di alcuna ragione o credito verso il minore.

15. Si applicano all'amministratore di sostegno, in quanto compatibili, le disposizioni di cui agli articoli (...) da 374 a 388.

16. Il tutore non può senza l'autorizzazione del giudice tutelare:1) acquistare beni, eccettuati i mobili necessari per l'uso del minore, per la economia domestica e per l'amministrazione del patrimonio; 2) riscuotere capitali, consentire alla cancellazione di ipoteche o allo svincolo di pegni, assumere obbligazioni, salvo che queste riguardino le spese necessarie per il mantenimento del minore e per l'ordinaria amministrazione del suo patrimonio; 3) accettare eredità o rinunciarvi, accettare donazioni o legati soggetti a pesi o a condizioni; 4) fare contratti di locazione d'immobili oltre il novennio o che in ogni caso si prolunghino oltre un anno dopo il raggiungimento della maggiore età; 5) promuovere giudizi, salvo che si tratti di denunzie di nuova opera o di danno temuto, di azioni possessorie o di sfratto e di azioni per riscuotere frutti o per ottenere provvedimenti conservativi.

17. Il tutore non può senza l'autorizzazione del tribunale: 1) alienare beni, eccettuati i frutti e i mobili soggetti a facile deterioramento; 2) costituire pegni o ipoteche; 3) procedere a divisioni o promuovere i relativi giudizi; 4) fare compromessi e transazioni o accettare concordati. L'autorizzazione è data su parere del giudice tutelare.

18. En el proyecto inicial se incluía un tercer presupuesto por el que entraría en juego la amministrazione di sostegno: el hecho de contar con una edad avanzada - vid. artículo 3 de la iniciativa parlamentare n. 2189 della XIV legislatura-. Sin embargo, finalmente se optó por suprimir dicho presupuesto, algo que a mi modo de ver resulta acertado, pues el hecho de contar con una edad avanzada, si no va acompañado de la presencia de determinadas enfermedades o discapacidades, no conlleva per se incapacidad para gestionar los propios intereses (en este mismo sentido: Napoli, 2002: 2).

19. La persona che, per effetto di una infermità ovvero di una menomazione fisica o psichica, si trova nella impossibilità, anche parziale o temporanea, di provvedere ai propri interessi, può essere assistita da un amministratore di sostegno(...).

20. Aunque es cierto que en el caso de enfermedades o discapacidades físicas podría recurrirse a otras figuras previstas en el ordenamiento jurídico italiano para que el beneficiario pudiera actuar en el tráfico jurídico - ej. al contrato de mandato-, el legislador ha preferido extender la 
permanente, y 2) que a causa de ella se vea afectada la autonomía de la persona de forma parcial o completa para la gestión de sus propios intereses (Cass., 4 febbraio 2014 y Trib. Modena, 5 febbraio 2016 ${ }^{21}$ ). Ello ha llevado a jurisprudencia (Cass., 11 settembre 2015; y Trib. Modena 4 maggio 2017) y doctrina (Ciliberti et al., 2008: 1) a considerar que puede recurrirse a la figura de la amministrazione di sostegno tanto en supuestos de patologías graves como leves.

Una de las principales dificultades que se han planteado en la práctica deriva de la coexistencia de tres figuras de protección de las personas privadas de autonomía, y consiste precisamente en determinar cuándo debe acudirse a la figura de la amministrazione di sostegno, y cuándo a las figuras preexistentes de la interdizione y la inabilitazione. Y es que el legislador no ha abordado esta cuestión, lo que le ha costado la crítica de un sector de la doctrina italiana (Curatola, 2006: 1; Vaccaro, 2012: 4).

Entre la amministrazione di sostegno y la inabilitazione, la jurisprudencia ha considerado que se aplicará esta última figura cuando, a tenor de las circunstancias, se estime que el sujeto necesita asistencia para la realización de prácticamente todos los actos de extraordinaria administración, mientras que se recurrirá a la amministrazione di sostegno cuando pueda realizar por sí mismo la mayor parte de dichos actos, necesitando asistencia únicamente para algunos actos puntuales (Corte Cost., 9 dicembre 2005).

Los problemas se han planteado especialmente en la delimitación de los presupuestos de la amministrazione di sostegno y de la interdizione, pues en algunos casos parecen coincidir totalmente, como ha venido advirtiendo la doctrina (Bembo, 2005: 2; Valle, 2007: 1; Masoni, 2007: 2; Ciliberti et al., 2008: 1; Cocuccio, 2010: 1; Giorgianni, 2013: 2; lurilli, 2017: 9). En el caso de la interdizione se exige que la patología tenga una cierta habitualidad o duración en el tiempo y que imposibilite completamente al sujeto -artículo 414 del Codice Civile - (Cass., 20 novembre 1985), mientras que el ámbito de aplicación de la amministrazione di sostegno es más amplio: incluye tanto los supuestos de enfermedades temporales y los de aquellas que inhabilitan solo parcialmente al sujeto (Cass., 12 giugno 2006), como los de enfermedades o discapacidades prolongadas que impiden totalmente al afectado por ellas gestionar por sí mismo sus propios intereses (Bembo, 2005: 3; Masoni, 2007: 1-2; Curatola, 2006: 5-7), coincidiendo en este segundo caso con el supuesto de hecho de la interdizione.

En un primer momento, un sector de la jurisprudencia (Trib. Monza, 6 luglio 2004; Trib. S. Maria Capua a Vetere, 30 agosto 2004; Trib. Bari, 5 ottobre 2004; Trib. Catania, 26 ottobre 2004; Trib. Milano, 11 novembre 2004; Trib. Ancona, 11 gennaio 2005; Trib. Ancona, 17 marzo 2005; Trib. Milano, 21 marzo 2005; y Trib. Bologna, 27 febbraio 2006) y de la doctrina (Masoni, 2007: 2) consideraron que el criterio que debía seguirse para determinar si debe acudirse a la figura de la amministrazione di sostegno o a la de la interdizione era el grado o intensidad de la discapacidad del sujeto ${ }^{22}$. El problema es que, como hemos visto, la amministrazione di sostegno también cabe en los supuestos de enfermedades o discapacidades graves, pues el propio

protección que otorga la amministrazione di sostegno también a aquellas personas que tienen enfermedades o discapacidades de carácter físico (Di Marzio, 2005: 2; y Marcoz, 2005: 3).

21. En el caso en cuestión, el Tribunale considera que, pese a que el individuo presenta una cierta discapacidad psíquica, ello no está afectando a su autonomía para gestionar sus propios intereses, ya que está bien insertado en la red social del lugar de residencia y realiza con autonomía las distintas actividades de la vida cotidiana. Por ello, entiende que no concurre el segundo de los presupuestos de la amministrazione di sostegno y rechaza su establecimiento.

22. A modo de ejemplo, la Sentencia del Tribunale di Milano de 21 de marzo de 2005 rechazó el establecimiento de un régimen de amministrazione di sostegno que habían solicitado unos padres cuya hija tenía graves enfermedades psicofísicas, por considerar que la interdizione era la medida más adecuada y eficaz para la protección de sus intereses. 
artículo 404 del Codice Civile admite su establecimiento cuando la patología impida totalmente al sujeto la gestión de sus intereses (Bembo, 2005: 3). Ello pone en entredicho la utilidad del mencionado criterio (Trib. Roma, 28 gennaio 2005).

La cuestión ha sido resuelta por la Corte Costituzionale, que ha manifestado que el criterio para determinar si debe recurrirse a la amministrazione di sostegno o a la interdizione no es cuantitativo sino cualitativo, en el sentido de que lo determinante no es la intensidad de la discapacidad, sino la mayor idoneidad de cada figura para adecuarse a las exigencias del sujeto (Corte Cost., 9 dicembre 2005). Esta doctrina sería seguida por la Corte di Cassazione (Cass., 12 giugno 200623; Cass., 22 aprile 2009; Cass., 1 marzo 2010; Cass. 26 ottobre 2011 y Cass., 11 settembre 2015) y por la jurisprudencia menor (Trib. Teramo, 14 febbraio 2013 ; Trib. Lecce, 3 agosto 2016; y Trib. Torino 22 settembre 2017). En este sentido, la Corte di Cassazione ha señalado que la elección entre una u otra figura dependerá de aspectos tales como la mayor o menor complejidad de la actividad que deba desempeñar la persona que va a representar o asistir a la persona con discapacidad o la magnitud del patrimonio de este último (Cass., 26 ottobre 2011 y Cass., 26 luglio 2013). Así, ha considerado que la figura idónea es la admministrazione di sostegno cuando se trata de realizar operaciones sencillas - ej. gestión de la pensión que recibe el beneficiario- ${ }^{24}$, mientras que procederá la interdizione cuando resulte necesario desempeñar funciones de cierta complejidad (Cass., 1 marzo 201025; Cass., 26 ottobre 2011; y Trib. Torino 22 settembre 2017). Sin perjuicio de ello, ha señalado que también podrá ser tenida en cuenta la gravedad o duración de la enfermedad, pero no en sustitución del anterior criterio, sino como complemento del mismo (Cass., 22 aprile 2009).

Como puede deducirse de lo anterior, el juez cuenta con un amplio margen de discrecionalidad para optar entre la amministrazione di sostegno y los otros dos instrumentos de protección que ofrece el ordenamiento italiano -interdizione e inabilitazione- (Bembo, 2005: 3).

Pero el asunto se complica todavía más si tenemos en cuenta que, como vamos a ver en el siguiente apartado, la competencia para establecer el régimen de amministrazione di sostegno y el de interdizione e inabilitazione no corresponde al mismo órgano judicial. Ello supone que el juez debe limitarse a valorar si la medida que le compete adoptar es la más adecuada para el supuesto concreto y, en caso contrario, poner en marcha el mecanismo para el traslado del expediente al órgano competente.

En cualquier caso, lo cierto es que la interdizione y la inabilitazione han pasado a jugar un papel residual, pues en la práctica se reservan para aquellos supuestos en los que se considera que la amministrazione di sostegno, pese a su flexibilidad y posibilidades de adaptación, no permite una protección suficiente del sujeto carente de autonomía y de su patrimonio (Corte Cost., 9 dicembre 2005; Corte Cost., 9 dicembre 2009; y Trib. Teramo, 14 febbraio 2013; Trib. Torino 22 settembre 201726; Vitulo, 2004: 5, Bembo, 2005: 2;

23. «(...) l'ambito di applicazione dell'amministrazione di sostegno va individuato non già al diverso, e meno intenso, grado di infermità o di impossibilità di attendere ai propri interessi del soggetto carente di autonomía, ma piuttosto alla maggiore capacità di tale strumento di adeguarsi alle esigenze di detto soggetto, in relazione alla sua flessibilità e dalla maggiore agilità della relativa procedura applicativa".

24. Vid. también: Trib. Benevento, 18 gennaio 2019, en la que se rechaza establecer un régimen de interdizione pese a que el sujeto en cuestión tenía una discapacidad severa. El motivo fue precisamente le escasa entidad de los actos que debía realizar, y que se limitaban al cobro de la pensión de la que era beneficiario. En términos semejantes: Trib. Vercelli, 31 ottobre 2014.

25. «un'attività minima, estremamente semplice, e tale da non rischiare di pregiudicare gli interessi del soggetto, vuoi per la scarsa consistenza del patrimonio disponibile, vuoi per la semplicità delle operazioni da svolgere (attinenti, ad esempio, alla gestione ordinaria del reddito da pensione)(...) corrisponderà l'amministrazione di sostegno»(...) «si potrà ricorrere all'interdizione quando si tratta di gestire un'attività di una certa complessità(...)». 26. En el caso enjuiciado por el Tribunale di Torino, se considera preferible la interdizione a la amministrazione di sostegno porque la persona en cuestión sufría un déficit neurocognitivo orgánico degenerativo con un grave deterioro cognitivo. Al respecto, el Tribunale argumenta que la 
Petrone, 2006: 7; Curatola, 2006: 2; Venchiarutti, 2007: 2-4; Valle, 2007: 3; Nardelli, 2008: 1; Ciliberti, 2008 : 1; Cocuccio, 2010: 2; Buffone, 2011: 5).

Por lo demás, la amministrazione di sostegno es incompatible con cualquiera de las otras dos figuras de protección -interdizione e inabilitazione- (Vitulo, 2004: 4). Prueba de ello es que si la persona en cuestión estuviera sujeta a un régimen de interdizione o inabilitazione, junto a la solicitud del establecimiento de la amministrazione di sostegno deberá instarse la revocación de dicho régimen -artículo 406.2 del Codice Civile- ${ }^{27}$. Además, los efectos de la amministrazione di sostegno se posponen hasta el momento en el que se publique la sentencia por la que se revoque la interdizione o la inabilitazione - artículo 405.3 del Codice Civile-28.

\section{c) Órgano competente para el establecimiento de la interdizione, la inabilitazione y la amministrazione di sostegno}

La competencia en materia de interdizione e inabilitazione viene atribuida a un tribunale ordinario de composición colegiada (Marcoz, 2005: 7; y Torroni, 2006: 1), mientras que la competencia para establecer el régimen de amministrazione di sostegno corresponde al giudice tutelare del lugar en el que el beneficiario tenga su residencia o domicilio - artículo 404 del Codice Civile-29.

Esta distribución competencial puede provocar problemas de coordinación entre los distintos órganos judiciales (Trib. Venezia, 24 settembre 2004; Passanante, 2006a: 9), ya que, en determinados casos, el expediente deberá trasladarse de un órgano a otro (Passanante, 2006b: 1):

Por ejemplo, en el proceso por el que se pone fin a la interdizione o inabilitazione, puede considerarse conveniente nombrar a un amministratore di sostegno. Al respecto, el artículo 429.3 del Codice Civile establece que el tribunale, de oficio o a instancia de parte, transmitirá el asunto al giudice tutelare ${ }^{30}$.

También puede ocurrir lo contrario, es decir, que en el proceso por el que se extingue la amministrazione di sostegno se estime oportuno que se aplique al sujeto el régimen de la interdizione o el de la inabilitazione. En tal caso, el artículo 413.4 del Codice Civile prevé que si el giudice tutelare considera que debe promoverse un juicio de interdizione o inabilitazione, informará al ministerio público para que este interponga la correspondiente acción ante el tribunal competente ${ }^{31}$.

\footnotetext{
amministrazione di sostegno sería insuficiente para proteger adecuadamente al sujeto, ya que es totalmente incapaz de atender por sí sola las necesidades de la vida cotidiana y de colaborar con un amministratore di sostegno en la gestión de sus propios intereses.

27. Se il ricorso concerne persona interdetta o inabilitata il medesimo è presentato congiuntamente all'istanza di revoca dell'interdizione o dell'inabilitazione davanti al giudice competente per quest'ultima.

28. Se l'interessato è un interdetto o un inabilitato, il decreto è esecutivo dalla pubblicazione della sentenza di revoca dell'interdizione o dell'inabilitazione.

29. (...) nominato dal giudice tutelare del luogo in cui questa ha la residenza o il domicilio.

30. Se nel corso del giudizio per la revoca dell'interdizione o dell'inabilitazione appare opportuno che, successivamente alla revoca, il soggetto sia assistito dall'amministratore di sostegno, il tribunale, d'ufficio o ad istanza di parte, dispone la trasmissione degli atti al giudice tutelare.

31. Il giudice tutelare provvede altresì, anche d'ufficio, alla dichiarazione di cessazione dell'amministrazione di sostegno quando questa si sia rivelata inidonea a realizzare la piena tutela del beneficiario. In tale ipotesi, se ritiene che si debba promuovere giudizio di interdizione o di inabilitazione, ne informa il pubblico ministero, affinché vi provveda(...).
} 
Así mismo, puede suceder que durante el procedimiento de declaración de la interdizione o de la inabilitazione se ponga de manifiesto que la medida más adecuada sería la amministrazione di sostegno. Se trata de un supuesto que no está expresamente previsto en el Codice Civile, pero jurisprudencia (Trib. Bologna, 8 marzo 2005) y doctrina (Passanante, 2006b: 1-2) han coincidido en señalar que el tribunale debe remitir el asunto al giudice tutelare, por ser este el órgano competente para establecer la amministrazione di sostegno.

Por último, también puede ocurrir que en el proceso de nombramiento del amministratore di sostegno se revele que esta medida es insuficiente para proteger adecuadamente a la persona con discapacidad y se considere más oportuno acudir a un proceso de interdizione o inabilitazione, una posibilidad sobre la que el Codice Civile italiano tampoco se pronuncia. Al respecto, jurisprudencia (Trib. Torino, 22 maggio 2004 y Trib. Milano, 21 marzo 2005) y doctrina (Passanante, 2006b: 2) han considerado que ha de aplicarse analógicamente lo dispuesto en el artículo 413.4 del Codice Civile, y, por tanto, el giudice tutelare deberá ponerlo en conocimiento del ministerio público para que este proceda a interponer la correspondiente acción ante el tribunal competente.

\section{Influencia de la normativa italiana en la reforma prevista en España a través del anteproyecto de ley por la que se reforma la legislación civil y procesal en materia de discapacidad: principales semejanzas y diferencias}

Como he avanzado en el apartado introductorio, el artículo 12 de la Convención sobre los derechos de las personas con discapacidad de 2006 establece que las personas que tienen una discapacidad tienen capacidad jurídica en igualdad de condiciones con las demás en todos los aspectos de la vida, y obliga a los Estados parte a adoptar las medidas pertinentes para proporcionar acceso a las personas con discapacidad al apoyo que puedan necesitar en el ejercicio de su capacidad jurídica.

Con el fin adaptar la normativa a dichas prescripciones, el gobierno de España se puso manos a la obra hay que reconocer que con cierta demora - y el 21 de septiembre de 2018 presentó el anteproyecto de ley por la que se reforma la legislación civil y procesal en materia de discapacidad, que, de prosperar, supondrá un nuevo paradigma en materia de protección de las personas con discapacidad en nuestro país.

Tradicionalmente, la protección de las personas con discapacidad se ha llevado a cabo por parte de nuestro ordenamiento a través de las figuras de la tutela, de la curatela y del defensor judicial -artículo 215 de la actual redacción del Código Civil-. Sin embargo, la reforma proyectada pretende introducir importantes novedades en el sistema clásico: entre otras cuestiones, propone suprimir la tutela ${ }^{32}$ - que quedaría limitada a los menores de edad no emancipados que se encuentren en situación de desamparo o que no estén sujetos a patria potestad ${ }^{33}$ - y dotar a la curatela de una nueva configuración, convirtiéndola en la principal medida de apoyo para las personas con discapacidad ${ }^{34}$. Así, la protección de las personas con discapaci-

32. Así como la patria potestad prorrogada y la patria potestad rehabilitada - vid. Exposición de motivos del anteproyecto-

33. Vid. artículo 1.19 del anteproyecto, en la redacción que pretende dar al artículo 199 del Código Civil. Vid. también: Exposición de motivos del anteproyecto.

34. Vid. Exposición de motivos del anteproyecto. 
dad pasará a articularse en torno a tres instrumentos: la guarda de hecho - que se referirá a aquellos casos en los que una persona ejerce el apoyo de otra con discapacidad, sin que existan medidas voluntarias o judiciales que se estén aplicando eficazmente-, la curatela - que se aplicará a quienes precisen el apoyo de modo continuado - y el defensor judicial - que procederá cuando la necesidad de apoyo se requiera de forma ocasional $-{ }^{35}$.

Como he anticipado, es probable que el legislador español se haya inspirado en la normativa italiana para establecer este nuevo sistema de protección, puesto que existen importantes puntos de conexión con dicho ordenamiento. El aspecto en el que resulta más evidente la influencia del derecho italiano en el anteproyecto de ley por la que se reforma la legislación civil y procesal en materia de discapacidad es el relativo a la nueva configuración con la que pretende dotar a la curatela, y que parece claramente inspirada en la amministrazione di sostegno italiana. No es vano, al igual que ocurre con la amministrazione di sostegno, la nueva configuración de la curatela tiene el propósito de sacrificar lo menos posible la capacidad del beneficiario para actuar en el tráfico jurídico ${ }^{36}, \mathrm{y}$, a tal fin, gozará de una enorme flexibilidad, admitiendo funciones tanto de representación como de asistencia y acomodando la actuación del curador a las necesidades particulares de la persona necesitada de apoyo ${ }^{37}$. Téngase en cuenta, no obstante, que sólo en situaciones donde el apoyo no pueda darse de otro modo, podrá concretarse en la representación o sustitución en la toma de decisiones $^{38}$.

En cuanto al contenido concreto de la nueva curatela, de modo semejante a lo que ocurre con la amministrazione di sostegno, deberá venir perfectamente determinado en la resolución que la establezca, que recogerá de manera precisa los actos en los que el curador deba prestar asistencia o ejercer la representación. Ya hemos visto que en el caso del derecho italiano dicha resolución recibe el nombre de Decreto di nomina, siendo competente para su adopción el giudice tutelare del lugar en el que el beneficiario tenga su residencia o domicilio. En nuestro derecho, estará constituida por la sentencia dictada por el Juzgado de Primera Instancia del lugar en el que resida la persona a la que se refiera la solicitud, por ser este el tribunal competente para establecer la curatela ${ }^{39}$. Por tanto, se huye de regímenes tipo y se trata de crear un proyecto personalizado para atender a las necesidades específicas del sujeto necesitado de protección. Así, tanto en uno como en otro caso, la persona con discapacidad conserva la capacidad para realizar por sí misma todos los actos que, de acuerdo a lo previsto en la correspondiente resolución, no requieran de representación o asistencia.

Y, en ambos casos, cuando la persona necesitada de apoyos realice actos que contravengan lo dispuesto en la mencionada resolución, dichos actos serán anulables. Ahora bien, en el derecho italiano, la acción para solicitar la anulación del acto prescribe en el plazo de cinco años a contar desde el momento en el que cese la amministrazione di sostegno - artículo 412.1 del Codice Civile - ${ }^{40}-$, mientras que el anteproyecto prevé un plazo de caducidad - no de prescripción, como en el caso del ordenamiento italiano- de cuatro

35. Vid.artículo 1.21 del anteproyecto, en la redacción que pretende dar al artículo 249 del Código Civil

36. Vid.artículo 1.21 del anteproyecto, en la redacción que pretende dar al artículo 266 del Código Civil Vid. también: Exposición de motivos del anteproyecto.

37. Vid. artículo 1.21 del anteproyecto, en la redacción que pretende dar a los artículos 248, 266 y 267 del Código Civil.

38. Vid. Exposición de motivos del anteproyecto.

39. Vid. artículo 3.7 del anteproyecto, en la redacción que pretende dar al artículo 756 de la Ley de Enjuiciamiento Civil.

40. Gli atti compiuti dall'amministratore di sostegno in violazione di disposizioni di legge, od in eccesso rispetto all'oggetto dell'incarico o ai poteri conferitigli dal giudice, possono essere annullati su istanza dell'amministratore di sostegno, del pubblico ministero, del beneficiario o dei suoi eredi ed aventi causa. 
años, que comenzará a contar desde el momento en el que la persona con discapacidad deje de precisar apoyo para celebrar el contrato ${ }^{41}$. En todo caso, la acción de anulabilidad no podrá ejercitarse pasados cinco años desde la celebración del contrato.

Pero, junto a las similitudes expuestas, también existen algunas diferencias entre las normas italiana y española:

La primera es relativa a la tutela, una figura que ya hemos visto que el ordenamiento italiano conserva entre los instrumentos de apoyo para las personas con discapacidad. Nuestro legislador, sin embargo, ha optado por una solución distinta, y, a través del anteproyecto de ley por la que se reforma la legislación civil y procesal en materia de discapacidad, pretende eliminar la tutela de nuestro sistema de protección de las personas con discapacidad. Así se viene reclamando desde diversas instancias ${ }^{42}$, argumentando que el establecimiento de mecanismos de sustitución resulta contrario a la Convención sobre los derechos de las personas con discapacidad de 2006, y, en concreto, a su artículo 12.2, que señala que "los Estados partes reconocerán que las personas con discapacidad tienen capacidad jurídica en igualdad de condiciones con las demás en todos los aspectos de la vida". De hecho, esta es la interpretación que lleva a cabo el Comité sobre los Derechos de las Personas con Discapacidad a través de su Observación general № 1 (2014), aprobada en su undécimo periodo de sesiones (31 de marzo a 11 de abril de 2014) ${ }^{43}$, en la que señala que "la obligación de los Estados partes de reemplazar los regímenes basados en la adopción de decisiones sustitutivas por otros que se basen en el apoyo a la adopción de decisiones exige que se supriman los primeros y se elaboren alternativas para los segundos. Crear sistemas de apoyo a la adopción de decisiones manteniendo paralelamente los regímenes basados en la adopción de decisiones sustitutiva no basta para cumplir con lo dispuesto en el artículo 12 de la Convención”. También en la doctrina italiana se ha cuestionado que el mantenimiento de la tutela resulte acorde a la Convención sobre los derechos de las personas con discapacidad (Lenti, 2019: 90).

Sin embargo, a mi modo de ver, suscita dudas que del tenor literal del artículo 12.2 de la Convención quepa extraer que la existencia de mecanismos de representación y sustitución suponga per se una vulneración de los principios recogidos en la misma, por lo que considero que la interpretación que hace la Observación general N 1 (2014) del Comité sobre los Derechos de las Personas con Discapacidad puede resultar excesivamente rígida. Creo que también es cuestionable la equiparación que lleva a cabo entre capacidad jurídica y capacidad de obrar, pues considerar que todas las personas poseen capacidad de obrar en igualdad de condiciones contradice la realidad física, psicológica, biológica, jurídica y social (García Cantero, 2014: 88). $Y$ es que, no es el derecho el que crea la falta de capacidad de obrar de una persona, sino que este se limita a reconocer dicha situación y a establecer mecanismos para protegerle.

41. Vid.artículo 1.47 del anteproyecto, en la redacción que pretende dar al artículo 1301 del Código Civil.

42. Vid. por ejemplo la "Propuesta articulada de reforma del Código Civil y de la Ley de Enjuiciamiento Civil para su adecuación al artículo 12 de la Convención sobre los Derechos de las Personas con Discapacidad”, presentada por la Subcomisión de Expertos del Real Patronato sobre Discapacidad el 13 de junio de 2012 (disponible en: https://www.notariosyregistradores.com/AULASOCIAL/2013-PropuestaModificacionCCLEC. pdf, fecha última consulta: 15/02/2020). En el mismo sentido se pronuncian Vivas (2010: 571) y Cuenca (2018: 83-84). Incluso el Tribunal Supremo, a partir de su Sentencia de 29 de abril de 2009, comenzó a mostrarse reacio al establecimiento de mecanismos de representación y sustitución (posteriormente le seguirían otros pronunciamientos: ej. STS 11 otubre 2012 y STS 24 junio 2013, en las que el alto Tribunal se decanta por la curatela en vez de por la tutela). Ahora bien, se trata en todo caso de supuestos de incapacidad parcial o limitada, pues en los casos de incapacidad absoluta ha continuado estableciendo la tutela como la medida más adecuada.

43. Disponible en: https://www.notariosyregistradores.com/AULASOCIAL/2013-Propuesta ModificacionCCLEC.pdf, fecha última consulta: 05/05/2020). 
En este sentido, no se puede perder de vista que el espíritu y finalidad principal de la Convención es garantizar la protección de los derechos de las personas que tienen una discapacidad, y habrá supuestos en los que dicho objetivo sólo pueda alcanzarse a través de una protección más intensa en la que la representación o sustitución puedan adquirir alcance general. Como pone de manifiesto Mayor del Hoyo, "la modificación de la capacidad de obrar tiene como fundamento último la protección y el bien de la persona, sin que, en modo alguno, su dignidad se vea afectada negativamente, sino al contrario. E igual que no ofrece controversia en el ámbito del menor, no lo debe ofrecer entre los mayores con problemas de autogobierno porque existe identidad de razón" (Mayor del Hoyo, 2013: 22 y 23).

Hay que tener en cuenta, además, que los supuestos de discapacidad son muy variados y las personas que la tienen requerirán diferentes sistemas de apoyo, lo que exige diseñar mecanismos de protección que garanticen que todas las modalidades de discapacidad queden suficientemente atendidas (Martínez de Aguirre, 2019: 270). No obstante, el anteproyecto parece que está pensando casi exclusivamente en aquellos casos en los que se ha tenido, y se mantiene, una cierta capacidad de formar la voluntad y tomar decisiones (Martínez de Aguirre, 2019: 270) y considero que no prestan la suficiente atención a los casos en los que esa capacidad no existe o es muy limitada. Para estos supuestos, creo que lo razonable es que el ordenamiento prevea la posibilidad de establecer mecanismos de representación o sustitución que tengan alcance general, como ocurre en el caso de la tutela -ya se utilice esta denominación, o ya se prefiera optar por otra, como curatela representativa-. Así ha sido entendido también por la Asociación de Profesores de Derecho Civil, que ha realizado en fechas recientes una propuesta de Código Civil cuyos artículos 171-1 y ss., si bien abogan por la creación de un sistema flexible para la protección de las personas con discapacidad, proponen llevarlo a cabo a través de las tradicionales figuras de la tutela y de la curatela (Asociación de Profesores de Derecho Civil, 2018: 289 y ss.).

A mayor abundamiento, de no preverse mecanismos de representación y sustitución para aquellos casos en los que la persona con discapacidad carezca de la más mínima capacidad natural de entender y querer, habrá un alto riesgo de que los actos que realice puedan ser impugnados y considerados inválidos por ausencia de consentimiento. Ello compromete no solo la protección de la propia persona con discapacidad, sino también la seguridad del tráfico y la protección de terceros (Martínez de Aguirre, 2019: 262).

La segunda diferencia en la que me voy a detener es relativa al posible establecimiento de medidas preventivas por parte del interesado en previsión de una futura necesidad de apoyo. Se trata de una posibilidad que se recoge en el anteproyecto de ley por la que se reforma la legislación civil y procesal en materia de discapacidad, pero no en la normativa italiana - probablemente, debido a la fecha de la que data, anterior a la Convención-. Al respecto, de acuerdo con la redacción propuesta en el anteproyecto, son dos las medidas que puede establecer una persona en previsión de una futura situación de discapacidad: los poderes y mandatos preventivos y la denominada autocuratela.

En cuanto a los poderes y mandatos preventivos, en palabras de Amunátegui, pueden definirse como "una forma de autoprotección basada en el concepto de apoderamiento, como una institución voluntaria, tanto en su propia consideración como en sus efectos, y que permite su configuración en el modo y manera en que lo disponga el mandante" (Amunátegui, 2019: 147). En este sentido, el anteproyecto prevé que, quien otorgue un poder en favor de otra persona, podrá incluir una cláusula que estipule que el poder subsista si en el futuro se ve necesitado de apoyo en el ejercicio de su capacidad ${ }^{44}$. Además, podrá otorgar poder solo

44. Vid. artículo 1.21 del anteproyecto, en la redacción que pretende dar al artículo 254 del Código Civil. 
para el supuesto de que en el futuro se vea necesitado de apoyo en el ejercicio de su capacidad. En este caso, para acreditar que se ha producido la situación de necesidad de apoyo se estará a las previsiones del poderdante ${ }^{45}$.

Por tanto, se distinguen dos tipos de poderes (Pau, 2018: 10): el convencional, respecto al que se admite su subsistencia en caso de que el mandante llegue a precisar de medidas de apoyo en el futuro; y el que podemos denominar verdaderamente preventivo, que solo será eficaz en caso de que el mandante precise de apoyo para el ejercicio de su capacidad.

Creo que la introducción de esta posibilidad supone un acierto por parte de nuestro legislador, pues permite un mayor poder de autorregulación de la propia discapacidad, en la línea marcada por la Convención sobre los derechos de las personas con discapacidad de 2006. No obstante, sería conveniente que el anteproyecto desarrollara con mayor precisión las distintas vicisitudes que pueden plantearse en torno a este instrumento, y, concretamente, que determinara si nos encontramos en todo caso ante un poder de representación o si también puede serlo de mera asistencia. En cuanto a esta última posibilidad, creo que tendría un difícil anclaje en los conceptos de poder y de mandato que se vienen manejando en nuestra tradición jurídica (en el mismo sentido: Amunátegui, 2019: 150; en sentido contrario: García, 2018: 10). Cuestión distinta es que el contenido del mandato incluya determinados actos y no otros, en cuyo caso el mandatario solo sustituirá al mandante para dichos actos (Amunátegui, 2019: 150).

En cuanto a la autocuratela ${ }^{46}$, de acuerdo al anteproyecto, cualquier persona mayor de edad o menor emancipada, en previsión de tener una futura situación de discapacidad, podrá proponer el nombramiento o la exclusión de una o varias personas para el ejercicio de la función de curador. Aquí encontramos una diferencia notable con el ordenamiento italiano, pues además de que la normativa omite cualquier referencia a esta cuestión, la jurisprudencia italiana rechaza la posibilidad de que una persona con buena salud y con un estado suficiente de conciencia y lucidez pueda nombrar un amministratore di sostegno en previsión de futuros e inciertos acontecimientos relativos a su salud. Para que ello proceda, exige la presencia de situaciones patológicas en curso, es decir, que estén ya presentes y clínicamente comprobadas (Trib. Modena, 10 dicembre 2015).

Por último, cabe destacar la decidida apuesta del legislador español por el reforzamiento de la guarda de hecho ${ }^{47}$, otra importante diferencia con el derecho italiano. Pérez Monge define dicha figura "como el ejercicio, con respecto a menores o personas que pudieran precisar de una institución de protección y apoyo, de funciones propias de instituciones tutelares, con carácter de generalidad y permanencia, de su custodia o protección, o de administración de su patrimonio o gestión de sus intereses por personas que no son tutores, curadores ni defensores judiciales" (Pérez Monge, 2019: 321-322). Una de las principales características de este tipo de guarda es que carece de investidura judicial formal y se ejerce de facto - como su propio nombre indica-, y cabe entender que así lo seguirá siendo de prosperar la reforma proyectada en el anteproyecto ${ }^{48}$. En palabras de Pau, se trata "de que el guardador de hecho pueda realizar actos represen-

45. Vid. artículo 1.21 del anteproyecto, en la redacción que pretende dar al artículo 255 del Código Civil.

46. La autocuratela vendría a sustituir a la denominada autotutela. De hecho, a partir de la entrada en vigor de la nueva ley, las previsiones de autotutela se entenderán referidas a la autocuratela - vid. Disposición transitoria tercera del anteproyecto-.

47.Vid. artículo 1.21 del anteproyecto, en la redacción que pretende dar a los artículos 249 y 261 del Código Civil.

48. De hecho, así se deduce de la redacción que el artículo 1.21 del anteproyecto pretende dar al artículo 261 del Código Civil: "Quien viniere ejerciendo adecuadamente la guarda de hecho de una persona con discapacidad, continuará en el desempeño de su función, a menos que existan medidas de apoyo de naturaleza voluntaria o judicial que se estén aplicando eficazmente (...)". 
tativos concretos a través de autorizaciones judiciales también concretas, sin necesidad de que haya tenido lugar un previo procedimiento judicial de determinación de apoyos" (Pau, 2018: 24).

Por tanto, la intervención judicial tendrá lugar únicamente cuando resulte necesaria la celebración de actos de representación por parte del guardador como consecuencia de la ausencia de capacidad de la persona con discapacidad para adoptar las decisiones relativas a su persona y bienes. Al respecto, el juez constatará que la guarda de hecho se esté ejerciendo adecuadamente, establecerá las medidas de control que estime oportunas y dotará al guardador de legitimación para el ejercicio de determinadas facultades de actuación (Leciñena, 2019: 294). Con ello, el anteproyecto dota a la guarda de hecho de un régimen jurídico que facilita la legitimación del guardador para realizar actos en beneficio de la persona y, a su vez, establece el mecanismo de control necesario para garantizar el correcto desempeño de su función (Pereña, 2018: 7). 
Referencias bibliográficas

Amunátegui, C. de (2019). "El protagonismo de la persona con discapacidad en el diseño y gestión del sistema de apoyo", en S. de Salas y M. V. Mayor (dirs.), Claves para la adaptación del ordenamiento jurídico privado a la Convención de Naciones Unidas en materia de discapacidad. Valencia: Tirant lo Blanch.

Asociación de Profesores de Derecho Civil (2018). Propuesta de Código Civil. Madrid: Tecnos.

Balestra, L. (2005). “Gli atti personalissimi del beneficiario dell’amministrazione di sostegno”, Familia, 4-5, pp. 1-9.

Bembo, M. D. (2005). "Amministrazione di sostegno e interdizione: un rapporto difficile”. Giurisprudenza costituzionale, 6, pp. 1-6.

Buffone, G. (2011). "La protezione giuridica dell'adulto incapace: I'anziano e l'amministrazione di sostegno". Giurisprudenza di Merito, 12, pp. 1-14.

Buffone, G. (2013). “L'istituto dell'amministrazione di sostegno”. Giurisprudenza di Merito, 11, pp. 1-10.

Ciliberti, L. et al. (2008). "Amministrazione di sostegno, interdizione, inabilitazione: innovazione, continuità e convivenza degli istituti di tutela della persona non autonoma". Rivista Italiana di Medicina Legale, 3, pp. 1-11.

Cocuccio, M. (2010). "L'amministrazione di sostegno come srumento prioritario di protezione e progetto di sostegno della persona con disabilità". Diritto di Famiglia e delle Persone, 0, pp. 1-10.

Cordiano, A. (2011). "L'esercizio delle situazioni esistenziali del beneficiario dell'amministrazione di sostegno". Diritto di Famiglia e delle Persone, 4, pp. 1-25.

Cuenca, P. (2018). "Reflexiones sobre el Anteproyecto de reforma de la legislación civil española en materia de capacidad jurídica de las personas con discapacidad". Cuadernos Electrónicos de Filosofía del Derecho, 38, pp. 82-101. DOI: https://doi.org/10.7203/CEFD.38.13308

Curatola, C. (2006). "Le aree di operatività dell'amministrazione di sostegno in relazione all'interdizione ed all'inabilitazione". Rivista del Notariato, 4, pp. 1-7.

Di Marzio, M. (2005). "Forme moderne di tutela per i disabili - in campo l'amministrazione di sostegno". Diritto e Giustizia, 4-5, pp. 1-3.

Eramo, F. (2004). “L’amministrazione di sostegno”, Diritto di Famiglia e delle Persone, 2, pp. 1-7.

García Cantero, G. (2014). “¿Persons with disability vs. personas incapacitadas... o viceversa?: Inserción del art. 12 del Convenio de Nueva York de 2006, en el ordenamiento español”. Revista de Derecho Civil, 4(1), pp. 67106.

García, M.P. (2018). “Las medidas de apoyo de carácter voluntario, preventivo o anticipatorio". Revista de Derecho Civil, 3(5), pp. 26-60.

Giorgianni, M. F. (2013). “La flessibilità dell'amministrazione di sostegno”. Rivista del Notariato, 2, pp. 1-4.

lurilli, C. (2017). "L'amministrazione di sostegno: poteri e funzioni in tema di giochi, scomesse e ludopatia". Diritto di Famiglia e delle Persone, 1, pp. 1-18.

Lavedini, G. (2007). “Prime brevi considerazioni sul ruolo dell'ente locale nell'amministrazione di sostegno", Diritto di Famiglia e delle Persone, 1, pp. 1-11. 
Leciñena, A. (2019). "La guarda de hecho como institución de apoyo a las personas con discapacidad", en S. de Salas y M. V. Mayor (dirs.), Claves para la adaptación del ordenamiento jurídico privado a la Convención de Naciones Unidas en materia de discapacidad. Valencia: Tirant lo Blanch.

Lenti, L. (2019). "La implementación de la Convención de Naciones Unidas en el entorno europeo: la amministrazione di sostegno italiana", en Salas, S. de y Mayor, M.V. (dirs.): Claves para la adaptación del ordenamiento jurídico privado a la Convención de Naciones Unidas en materia de discapacidad. Valencia: Tirant lo Blanch.

Marcoz, G. (2005). "La nuova disciplina in tema di amministrazione di sostegno”, Rivista del Notariato, 1, pp. 523546.

Martínez de Aguirre, C. (2019). "Curatela y representación: cinco tesis heterodoxas y un estrambote”, en S. de Salas y M. V. Mayor (dirs.), Claves para la adaptación del ordenamiento jurídico privado a la Convención de Naciones Unidas en materia de discapacidad. Valencia: Tirant lo Blanch.

Mascolo, F. y Marcoz, G. (2005). "L'amministrazione di sostegno e l'impianto complessivo del Codice Civile". Rivista del Notariato, 6, pp. 1-20.

Masoni, R. (2007). "Amministrazione di sostegno ed interdizione, dal diritto al dovere di sostegno?”. Giurisprudenza di Merito, 1, pp. 1-10.

Masoni, R. (2009). "Consenso e dissenso del beneficiario dell'amministrazione di sostegno". Diritto di Famiglia e delle Persone, 1, pp. 1-8.

Masoni, R. (2013). "Annullabilità del contrato concluso dall'amministrazione di sostegno e regime giuridico applicabile”. Giurisprudenza di Merito, 1, pp. 1-5.

Masoni, R. (2016). "La cura personale nell'amministrazione di sostegno secondo la Cassazione Penale". Diritto di Famiglia e delle Persone, 3, pp. 1-8.

Masoni, R. (2017). "Il matrimonio del disabile psichico soggeto ad amministrazione di sostegno". Diritto di Famiglia e delle Persone, 3, pp. 1-7.

Mayor del Hoyo, M.V. (2013). Un nuevo modelo de protección de las personas con discapacidad. Cizur Menor: Aranzadi.

Naciones Unidas (2006). Convención sobre los Derechos de las Personas con Discapacidad. Nueva York: ONU.

Napoli, E. V. (2002). "Una terza forma d'incapacità d'agire? Note a margine di una proposta di legge sull'amministrazione di sostegno". Giustizia Civile, 9, pp. 1-5.

Nardelli, M. (2008). “Ll’amministratore di sostegno tra incerti conflitti e possibili soluzioni”. Giurisprudenza di Merito, 1, pp. 1-9.

Paradiso, M. (2005). "L'amministrazione di sostegno (leggendo il quaderno di familia)", Familia, 3, pp. 1-6.

Passanante, L. (2006a). "Profili processuali dell'amministrazione di sostegno”. Familia, 2, pp. 1-14.

Passanante, L. (2006b). “Raporti tra amministrazione di sostegno, interdizione e inabilitazione: profili processuali”. Giustizia Civile, 4-5, pp. 1-5.

Pau, A. (2018). "De la incapacitación al apoyo: el nuevo régimen de la discapacidad intelectual en el Código Civil”. Revista de Derecho Civil, 3(5): pp. 5-28.

Pereña, M. (2018). "La transformación de la guarda de hecho en el Anteproyecto de Ley". Revista de Derecho Civil, 3(5), pp. 61-83. 
Pérez Monge, M. (2019). "La guarda de hecho", en S. de Salas y M. V. Mayor (dirs.), Claves para la adaptación del ordenamiento jurídico privado a la Convención de Naciones Unidas en materia de discapacidad. Valencia: Tirant lo Blanch.

Petrone, N. (2006). "Coordinamento fra le misure di protezione delle persone prive di autonomía, revoca dell'inabilitazione e scelta fra l'interdizione e l'amministrazione di sostegno". Rivista del Notariato, 2, pp. 1-13.

Rocchio, F. y Liserre, A. (2017). Lezioni di diritto privato. $4^{\text {a }}$ ed. Milán: Giuffrè.

Serrao, E. (2006). "Il procedimento per la nomina dell'amministrazione di sostegno”, Giurisprudenza di Merito, 5, pp. 1-9.

Torroni, F. (2006). "Brevi note circa l'interferenza tra i procedimenti d'interdizione e di nomina dell'amministratore di sostegno". Rivista del Notariato, 2, pp. 1-5.

Vaccaro, G. E. (2012). "Sulla tutela dei cittadini attraverso l'istituto dell'amministrazione di sostegno". Diritto di Famiglia e delle Persone, 4, pp. 1-7.

Valle, C. (2007). “'L'amministrazione di sostegno nella prassi giurisprudenziale”. Giurisprudenza di Merito, 9, pp. $1-13$.

Venchiarutti, A. (2007). "Il discrimen tra amministrazione di sostegno e interdizione: dopo la Corte Costituzionale si pronuncia la Corte di Cassazione". Diritto di Famiglia e delle Persone, 1, pp. 1-7.

Vitulo, F. (2004). "L'amministratore di sostegno, il tutore, il curatore". Comunicación presentada en Forum: L'amministratore di sostegno, analisi e prospettive di un nuovo strumento giuridico a favore delle persone prive di autonomia, Bologna. Recuperado de http://www.grusol.it/informazioni/30-05-06.PDF

Vivas, I. (2010). "Libertad y protección de la persona vulnerable en los ordenamientos jurídicos europeos: hacia la despatrimonialización de la discapacidad". Revista de Derecho UNED, 7, pp. 561-595. DOI: https://doi. org/10.5944/rduned.7.2010.11037

Vivas, I. (2012). Un nuevo modelo de protección de las personas con discapacidad. Badajoz: FUTUEX - Fundación para la promoción y apoyo a las personas con discapacidad. 


\section{ANEXO}

Abreviaturas

App.: Corte d’Appello (Tribunal de Apelación italiano).

Cass.: Corte di Cassazione (Tribunal Supremo italiano).

Corte Cost.: Corte Costituzionale (Tribunal Constitucional italiano)

Trib.: Tribunale (Tribunal ordinario italiano).

Trib. Min.: (Tribunal de menores italiano).

Jurisprudencia citada

a) Tribunal Supremo español

STS 29 abril 2009

STS 11 octubre 2012

STS 24 junio 2013

\section{b) Corte Costituzionale italiana}

Corte Cost., 9 dicembre 2005

c) Corte di Cassazione italiana

Cass., 20 novembre 1985

Cass., 12 giugno 2006

Cass., 16 novembre 2007

Cass., 22 aprile 2009

Cass., 1 marzo 2010
Cass., 26 ottobre 2011

Cass., 26 luglio 2013

Cass., 4 febbraio 2014

Cass., 11 settembre 2015

\section{d) Otros tribunales italianos}

Trib. Torino, 22 maggio 2004

Trib. Monza, 6 luglio 2004

Trib. S. Maria Capua a Vetere, 30 agosto 2004

Trib. Venezia, 24 settembre 2004

Trib. Bari, 5 ottobre 2004

Trib. Catania, 26 ottobre 2004

Trib. Milano, 11 novembre 2004

Trib. Ancona, 11 gennaio 2005

Trib. Roma, 28 gennaio 2005

Trib. Ancona, 17 marzo 2005

Trib. Milano, 21 marzo 2005

Trib. Pinerolo, 13 dicembre 2005

Trib. Bologna, 2 gennaio 2006

Trib. Bologna, 27 febbraio 2006

Trib. Vercelli, 31 ottobre 2014

Trib. Genova, 17 dicembre 2015

Trib. Modena, 10 dicembre 2015

Trib. Modena, 5 febbraio 2016

Trib. Lecce, 3 agosto 2016

Trib. Modena 4 maggio 2017

Trib. Torino 22 settembre 2017

Trib. Benevento, 18 gennaio 2019 